\title{
A rare cause of infertility: Uterine isthmocele
}

\author{
Mustafa Koplay*, Nusret Seher, Emine Uysal and Hakan Cebeci \\ Department of Radiology, Medical Faculty, Selcuk University, Konya, Turkey
}

A 35-year-old female patient with a history of cesarean section 2 times applied to our hospital with the complaints of post-menstrual bleeding for the last 1 year and infertility. In pelvic MRI, in cesarean section scar localization in the anterior uterine corpus, $15 \times 10 \mathrm{~mm}$ in size, well-circumscribed hypointense cystic lesions were observed on T1-weighted images, and hyperintense cystic lesions were observed on T2-weighted images (Figure 1). A thinning was observed in the uterine parenchyma in the anterior neighborhood of this area. In patients who have the story of cesarean section in uterine cystic lesions, the uterine isthmocele should be considered in definitive diagnosis.

A 35-year-old female patient with a history of cesarean section 2 times applied to our hospital with the complaints of post-menstrual bleeding for the last 1 year. In the transvaginal ultrasonography (TVUSG), anechoic cystic formation was observed in the anterior of the uterine corpus, and pelvic MRI examination was performed on the patient. At MRI, in cesarean section scar localization in the anterior uterine corpus, $15 \times 10 \mathrm{~mm}$ in size, well-circumscribed hypointense cystic lesions were observed on T1-weighted images, and well-circumscribed hyperintense cystic lesions were observed on T2weighted images. A thinning was observed in the uterine parenchyma in the anterior neighborhood of this area but parametrial fat planes were normal. Uterine isthmocele was diagnosed through imaging findings [1]

Uterine isthmocele is a diverticular cystic formation resulting from cesarean scar, incidence of which increases with the increase of cesarean numbers and imaging techniques in recent years [2]. The reason is unknown exactly. Previous cesarean section is the greatest risk factor and the number of cesarean section increases the risk [2]. Patients most frequently apply with symptoms such as postmenstrual
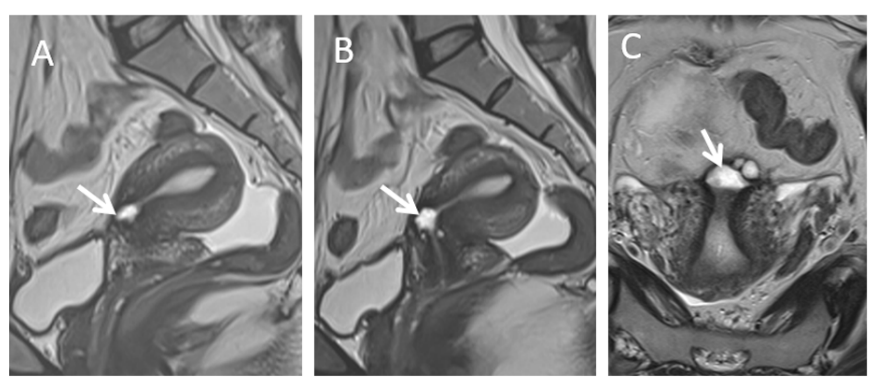

Figure 1. Cystic lesions

bleeding and infertility [3]. Uterine scar defects can be detected in \%4060 of TVUS and hysterosalpingography (HSG). In suspected cases, MRI is very useful for uterine isthmocele. In patients who have the story of cesarean section and who apply with symptoms such as postmenstrual bleeding and infertility, in uterine cystic lesions, the uterine isthmocele should be considered in definitive diagnosis and MRI is quite useful in diagnosis.

\section{References}

1. Drouin O, Bergeron T, Beaudry A, Demers S, Roberge S, et al. (2014) Ultrasonographic Evaluation of Uterine Scar Niche before and after Laparoscopic Surgical Repair: A Case Report. AJP Rep 4: e65-8. [Crossref]

2. Xie H, Wu Y, Yu F, He M, Cao M, et al. (2014) A comparison of vaginal surgery and operative hysteroscopy for the treatment of cesarean-induced isthmocele: a retrospective review. Gynecol Obstet Invest 77: 78-83. [Crossref]

3. Sipahi S, Sasaki K, Miller CE (2017) The minimally invasive approach to the symptomatic isthmocele - what does the literature say? A step-by-step primer on laparoscopic isthmocele - excision and repair. Curr Opin Obstet Gynecol 29: 257-265. [Crossref]
Copyright: (C2018 Koplay M. This is an open-access article distributed under the terms of the Creative Commons Attribution License, which permits unrestricted use, distribution, and reproduction in any medium, provided the original author and source are credited.
Correspondence to: Mustafa Koplay, Department of Radiology, Medical Faculty, Selcuk University, The Central Campus, 42075, Konya, Turkey, Tel: +90-3322243800- 44939; E-mail: koplaymustafa@hotmail.com

Received: February 02, 2018; Accepted: February 19, 2018; Published: February 22,2018 